\title{
A Recursive Cost-Based Approach to Fracturing
}

by Shangliang Jiang

\section{Research Project}

Submitted to the Department of Electrical Engineering and Computer Sciences, University of California at Berkeley, in partial satisfaction of the requirements for the degree of Master of Science, Plan II.

Approval for the Report and Comprehensive Examination:

\section{Committee:}

Professor Avideh Zakhor

Research Advisor

Date

*******

Professor Andrew R. Neureuther

Second Reader

Date 


\begin{abstract}
In microlithography, mask patterns are first fractured into trapezoids and then fabricated with a variable shaped beam writing machine. The efficiency and quality of the writing process is determined by the trapezoid count and external slivers. Slivers are trapezoids with width less than $\epsilon$, a parameter determined by the mask-writing tool. External slivers are slivers whose length is along the boundary of the polygon. External slivers have a large impact on critical dimension $(\mathrm{CD})$ variability and should be avoided. The shrinking $\mathrm{CD}$, increasing polygon density, and increasing use of resolution enhancement techniques have raised new challenges to control the trapezoid number and external sliver length. In this paper, we propose a recursive cost-based algorithm for fracturing which takes into account external sliver length as well as trapezoid count. We start by defining the notion of Cartesian convexity for rectilinear polygons. We then generate a grid-based sampling as a representation for fracturing. From these two ideas we develop two recursive algorithms; the first one utilizes a natural recurrence while the second one utilizes a more complex recurrence. Under Cartesian convexity conditions, the second algorithm is shown to provide optimal solutions; however, it has a drastically increased runtime as compared with the natural recurrence. Our simulations demonstrate the natural recurrence algorithm to produce significantly less sliver length than a commercially available fracturing tool by up to $60 \%$ in terms of external sliver length without increasing the polygon count.
\end{abstract}




\section{Contents}

1 Introduction $\quad 5$

2 Basics of Fracturing $\quad 7$

2.1 Minimum Cardinality Rectangle Partition . . . . . . . . . . . . . . . 7

2.2 Valid Fracturing . . . . . . . . . . . . . . . . . . 7

3 Fracturing Problem Formulation $\quad 9$

4 Proposed Algorithm 11

4.1 Representation of Rectilinear Polygons . . . . . . . . . . . . . . . . . . 11

4.2 Generating Costs for Rectangles within a Fracture . . . . . . . . . . . . . . 13

4.3 Solving a Cartesian Convex Polygon with a Natural Recurrence . . . . . . . . . . 14

4.4 Solving a Cartesian Convex Polygon with an Optimal Recurrence . . . . . . . . 15

4.5 Implementation of Recurrences using Dynamic Programming . . . . . . . . . . . 18

4.6 Nonconvex Polygons . . . . . . . . . . . . . . . . . . . . . . . . 19

5 Experimental Results $\quad 20$

6 Conclusion and Future Work $\quad 23$

7 Acknowledgments $\quad 24$ 
A Proof of optimality for the pinwheel recurrence 


\section{Introduction}

In optical lithography, light emitted from an illumination system is transmitted through the mask and generates an image on the wafer. Mask writing is a significant step that affects the fidelity of the printed image on the wafer and critical dimension (CD) control. The mask is printed using a Variable Shaped Beam (VSB) mask writing machine. As a first step the mask pattern is fractured into numerous non-overlapping trapezoids. Subsequently these trapezoids are exposed by the VSB mask writing machine onto the mask.

The use of a VSB mask writing system places requirements on the resulting fracturing solution. These requirements are either hard constraints that cannot be violated, or soft constraints that complicate the mask writing process and should preferably be avoided[1, 2].

1. The mask pattern must be partitioned into a set of non-overlapping basic trapezoids.

2. The maximum linear size of a shot produced by current VSB mask writing machines is between $2 \mu m$ and $3 \mu m$ on the mask scale.

3. To have good critical dimension uniformity the cumulative length of external slivers should be minimized.

4. In order to minimize the shot count and hence writing time, the resulting number of trapezoids should be minimized.

The first two requirements are hard constraints as VSB writing technology cannot produce arbitrary shapes and sizes. Furthermore, the basic trapezoids must be non-overlapping as overlapped regions would result in excess exposure leading to increased variability. The last two requirements are soft; both the number of trapezoids and external sliver length should preferably be minimized to improve mask write time and CD uniformity.

M. Bloecker et al. have evaluated many metrics to quantify fracturing quality [5]. The authors proposed and demonstrated that shoreline or external sliver length is a suitable metric for evaluating fracturing quality as it closely correlates with manufacturability while being fast to evaluate. Features with width below a certain $\epsilon$, as determined by the VSB mask-writing tool, are called 
slivers. Slivers whose length is along the boundary of the layout polygon are called external slivers. As the shot is smaller the current density becomes more steep which adversely influences the shot placement. Thus slivers have large size variability, adversely affecting CD control.[3]. Fig. 1 demonstrates two possible fracturing options and a possible tradeoff: Fig. 1(a) has fewer shots, and one external sliver while Fig. 1(b) has an extra shot as compared to Fig. 1(a) but no slivers. Depending on the requirements, a different fracturing should be employed, in practice.

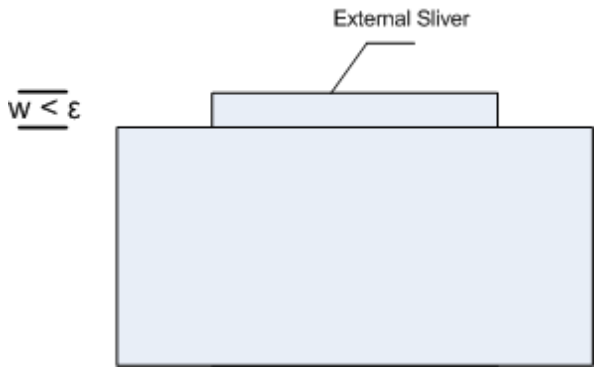

(a)

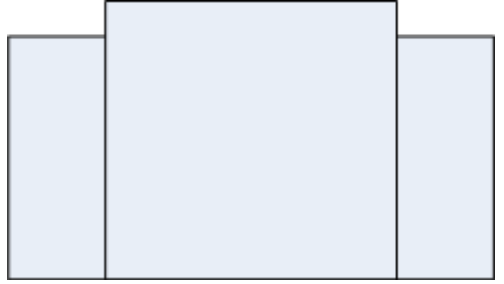

(b)

Figure 1: Two methods of fracturing the same polygon: (a) fracture solution with two polygons and one external sliver, (b) fracture solution with three polygons and zero external slivers.

C. Spence et al. have demonstrated that the number of shots is directly correlated with the write-time [4]. Furthermore, due to the small critical feature size, it is reasonable to assume that the shot count is equal to the number of post-fracture figures, namely the resulting trapezoids. From this it follows that the write-time is directly correlated to the number of post-fracture trapezoids and thus the latter needs to be minimized.

In this paper, we formulate the fracturing problem as an optimization problem by choosing a cost function that is the weighted sum of the number of rectangles and the external sliver length. We develop a new grid-based representation of a polygon and for this representation we propose two recursive algorithms for rectilinear polygons to solve the optimization formulation. A rectilinear polygon is a polygon whose corners form right angles. When the rectilinear polygon is restricted to be convex in the Cartesian directions, one of our proposed algorithms guarantees optimality. The other one is shown to be faster and empirically matches the results of the optimal algorithm.

In Section 2, we review results on fracturing that support our formulation choice. In Section 3, we describe our cost function and define Cartesian convexity for rectilinear polygons. In Section 4, we describe our proposed algorithm. In Section 5, we provide experimental results for our 
proposed algorithm as compared with a commercial solver. We conclude with possible directions for future work in Section 6.

\section{Basics of Fracturing}

\subsection{Minimum Cardinality Rectangle Partition}

T. Ohtsuki has given an exact $O\left(n^{5 / 2}\right)$ algorithm for partitioning a rectilinear polygon into the minimum number of rectangles[6] . The algorithm is based upon restricting partitioning rays to start only from concave corners and selecting the maximum disjoint set of chords. Chords are partitioning rays that start and terminate at concave rays. The maximum disjoint set of chords can be selected by finding a maximum independent set for a bipartite graph, which is equivalent to finding the maximum matching of the same bipartite graph, from König's Theorem[8]. This is achievable in $O\left(n^{5 / 2}\right)$ by solving the bipartite matching problem with the Hopcroft-Karp algorithm[9], where $n$ is the number of vertices.

This result provides two steps to minimize the total number of rectangles. First, the rays forming the partition should be restricted to those that start from concave corners. Second, the number of chords selected in the partitioning needs to be maximized.

\subsection{Valid Fracturing}

Kahng et al. have shown that a valid fracturing of a rectilinear polygon results in all interior points of the polygon satisfying 1 of 3 constraints shown in Fig 2[1,2]. A valid fracturing of a rectilinear polygon is a partition of the polygon that consists only of rectangles. We define a line segment as part of a line that is bounded by two end points. The 3 validity constraints on the interior points of the polygon are:

- The point has 0 line segments entering it, as shown in Fig. 2(a). 
- The point has 2 line segments entering that are in the same orientation; either both horizontal or both vertical. The vertical example is shown in Fig. 2(b).

- The point has 3 line segments entering it as shown in Fig. 2(c).

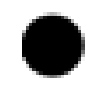

(a)

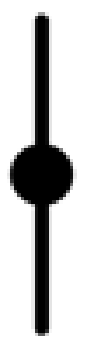

(b)

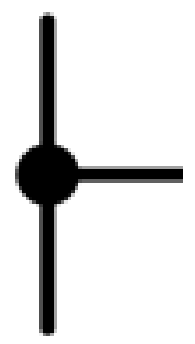

(c)

Figure 2: Valid internal points.

These three interior point constraints are the only valid configurations. The remaining two possibilities are invalid and are described below:

- The point has 1 line segment leaving it, as shown in Fig. 3(a). This cannot exist within a valid fracturing as this point and line segment cannot be part of a rectangle.

- The point has 2 line segments entering with different orientations; one is horizontal and the other is vertical. This configuration is invalid as the concave side cannot be part of a rectangle. An example is shown in Fig. 3(b).

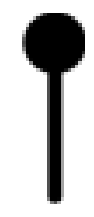

(a)

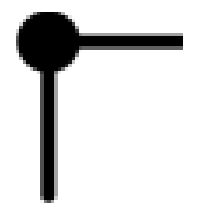

(b)

Figure 3: Invalid interior points.

As stated in Section 2.1, that to minimize the total number of rectangles resulting from the fracturing of a rectilinear polygon, only rays from concave corners should be used. In that case, 
all interior points of the polygon can be split into three groups. The first are interior points that do not lie on the path of any ray. These points never have any line segments passing through them. They always satisfy the constraint shown in Fig. 2(a) and do not need to be checked to confirm the fracturing is valid.

Next are the points that lie on the path of two rays. These interior points are marked by the intersections of the rays and need to be checked for the fracturing constraints in Fig. 2. However, there are only $O\left(n^{2}\right)$ such interior points, where $n$ is the number of concave corners. This follows since these interior points exist as the intersections of two rays from concave corners. These interior points are the only interior points upon which a line segment may terminate. A line segment must terminate upon a boundary to result in a valid fracturing; the boundary may be part of the polygon boundary or an earlier placed line segment. As interior points do not reside on the polygon boundary, only interior points at the intersection of two rays can be the location of a line segment termination.

Finally, there are interior points that lie along the path of only one ray. As line segments can only terminate at the points of intersections of two rays, these interior points cannot correspond to a terminated line segment. Therefore all interior points along the path of one ray must either satisfy the constraint in Fig. 2(a) i.e. no line segments pass through them or the constraint in Fig. 2(b) whereby a line segment passes through and continues in one of the Cartesian directions. Thus, with the restriction that line segments only terminate upon the intersection of two rays, these interior points do not need to be considered as they always satisfy the validity requirements for fracturing.

To summarize, the points for which validity of the fracturing is a concern are the $O\left(n^{2}\right)$ points that lie on the intersections of two rays, where $n$ is the number of concave corners. This motivates the representation proposed in Section 4.1.

\section{Fracturing Problem Formulation}

The VSB mask writing tool characteristics suggest two possibly conflicting goals in fracturing: the first is to minimize the number of resulting rectangles to limit shot count, and the second is 
to minimize the external sliver length for $\mathrm{CD}$ control. To capture both goals, we consider the following cost function:

$$
\#(\text { rectangles })+\lambda_{L} L(\text { external slivers })
$$

where $\lambda_{L}$ is a parameter chosen by the user to weigh the cost of external sliver length against the rectangle count and $L$ (external slivers) refers to the length of the external slivers. Heuristically, we found values of $\lambda_{L}$ calculated to $1 \mathrm{~nm}$ divided by the sliver parameter, $\epsilon$, to give good fracturings; providing improvements in external sliver length without increasing the rectangle count.

A rectilinear polygon, with more than 4 corners, must have concave vertices and cannot be a convex polygon. Nevertheless, we propose a definition of a Cartesian convexity for rectilinear polygons as a rectilinear polygon that is convex in the Cartesian directions. Mathematically, this can be written as:

$$
\begin{aligned}
& \left(x_{1}, y\right) \text { and }\left(x_{2}, y\right) \in P O L Y G O N \text { Then: } \forall t \in[0,1],\left((1-t) x_{1}+t x_{2}, y\right) \in P O L Y G O N \\
& \left(x, y_{1}\right) \text { and }\left(x, y_{2}\right) \in \text { POLYGON Then: } \forall t \in[0,1],\left(x,(1-t) y_{1}+t y_{2}\right) \in \text { POLYGON }
\end{aligned}
$$

The above equations state that if any two points that lie in a Cartesian convex rectilinear polygon can be joined by a line segment in a Cartesian direction, then all points in the line segment also lie within the polygon. This is equivalent to the property that any line in a Cartesian direction intersects the boundaries that are perpendicular to the line twice. Fig. 4 gives examples of a Cartesian convex and a non-convex rectilinear polygon.

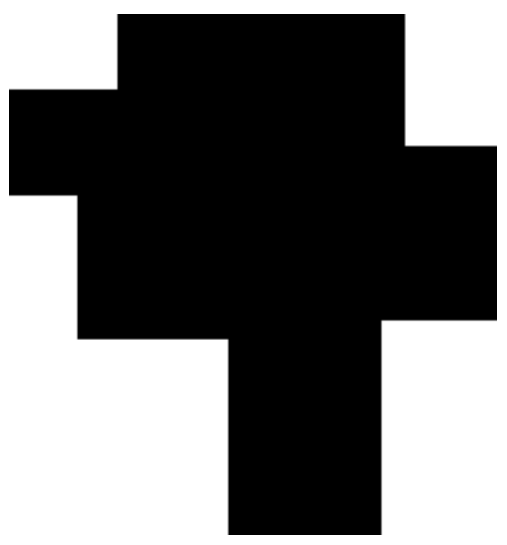

(a) Convex

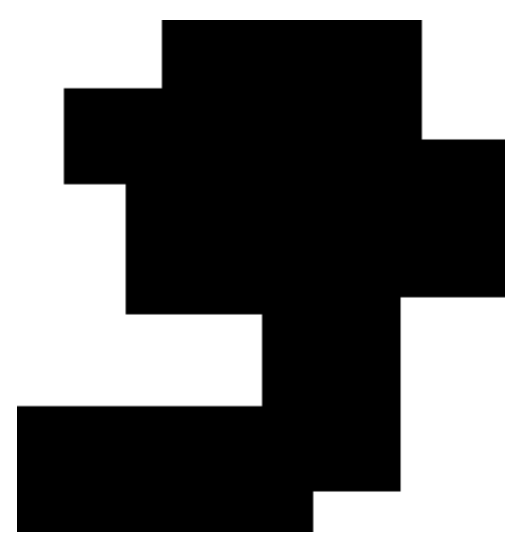

(b) Non-Convex

Figure 4: Comparison of Cartesian convex and non-convex rectilinear polygons. 
To summarize, the importance of this property is that any line in a Cartesian direction that lies within a Cartesian convex rectilinear polygon intersects the boundaries twice. This motivates our recurrences developed in Sections 4.3 and 4.4.

\section{Proposed Algorithm}

Our proposed algorithms in this paper are applicable to rectilinear polygons. For convenience, we use the term polygon to refer to rectilinear polygons for the remainder of this paper. Our algorithms apply 3 steps to generate a fracturing. Section 4.1 shows how to transform the polygon into a rectangular gridded array. Section 4.2 generates the costs for the internal rectangles. Sections 4.3 and 4.4 develop recursive algorithms used to find the lowest cost fracturing for Cartesian convex polygons. In Section 4.5 we describe the ideas behind dynamic programming and how they are used to maintain a polynomial runtime. In Section 4.6 we propose extensions to the algorithms to solve for the fracturing of non-Cartesian convex polygons.

\subsection{Representation of Rectilinear Polygons}

As stated in Section 2.1, rays should only be generated from concave corners to minimize rectangle count. Furthermore, in Section 2.2 we have shown that not all points within a polygon are relevant to the validity of a fracturing. The only relevant points are points where the edge constraints may be violated namely the corners, rays from each corner, and intersections of the rays with the boundary and with one another. We call the grid formed by these points the basic grid and use it to represent the polygon.

We propose the following representation towards dicretizing the problem. First, we embed the input polygon with a rectangle. Next, we generate all the relevant points by including all possible partitioning rays from every corner and noting the intersections. For the resulting basic grid we number the points with coordinates $(1,1)$ to $(x, y)$ as shown in Fig. 5.

The polygon may be represented by a binary array of size $[x \times y]$ where an entry is 0 if its 


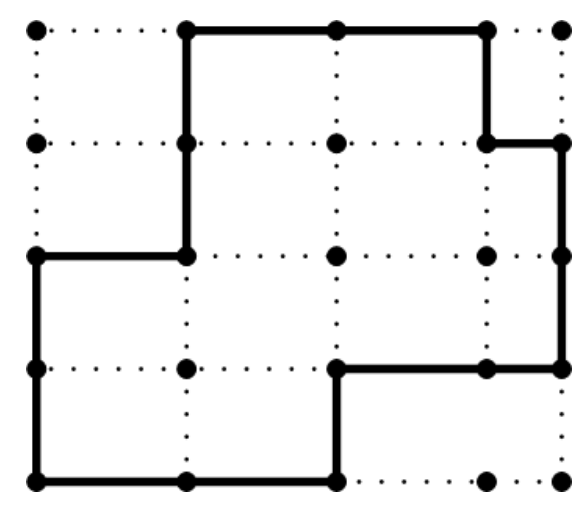

Figure 5: Embedding the polygon within a rectangle and generating the basic grid.

corresponding point is exterior to the polygon and 1 if the point is interior. $x$ corresponds to the number of discrete coordinates in the $x$-direction for all vertices and $y$ corresponds to the number of discrete coordinates in the $y$-direction. To represent a rectangular selection of the basic grid a pair of coordinates $[(a, b),(c, d)]$ may be used; the first coordinate $(a, b)$ refers to the top left corner while the second coordinate refers to the bottom right corner. This is shown in Fig. 6 where the marked rectangle is $[(2,3),(5,5)]$.

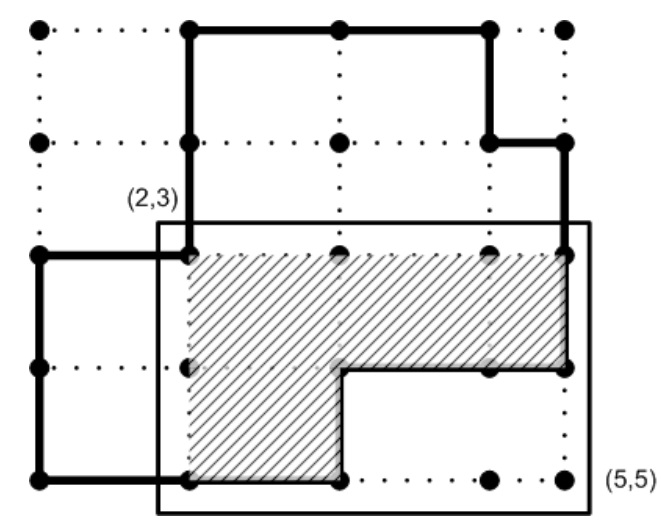

Figure 6: A representation of a sub-rectangle.

We define a sub-rectangle to be any rectangle that lies along the basic grid as shown in Fig. 6 . A sub-rectangle also represents the subsection of the polygon embedded within it. For example, in Fig. 6, the subsection of the polygon embedded with sub-rectangle $[(2,3),(5,5)]$ is shaded. This representation allows us to enumerate all possible sub-rectangles for a given rectilinear polygon. Specifically, the number of sub-rectangles is $O\left(n^{4}\right)$, where $n$ is the number of vertices in the original polygon. This is because the grid has $O\left(n^{2}\right)$ points and a sub-rectangle requires two points from the grid. 
The motivation behind this representation is that the cost function of any given sub-rectangle can be computed by considering all possible decompositions of the given sub-rectangle into smaller sub-rectangles and recursively repeating. At each step we select the decomposition that results in the lowest cost. A naive implementation results in an exponential runtime; however, in Section 4.5 we describe ways of applying the decomposition and storing costs to arrive at a tractable polynomial runtime.

\subsection{Generating Costs for Rectangles within a Fracture}

To solve the optimization problem we need to generate costs for each of the sub-rectangles. We assign each of the sub-rectangles a cost equal to the optimal partition of the subsection of the polygon enclosed by the sub-rectangle. For instance, the cost of the sub-rectangle, $[(2,3),(5,5)]$, in Fig. 7 is equal to the cost of the polygon enclosed, with vertices: $[(2,3),(5,3),(5,4),(3,4),(3,5),(2,5)]$. More generally, the four possible cases and resulting costs for sub-rectangles are described below:

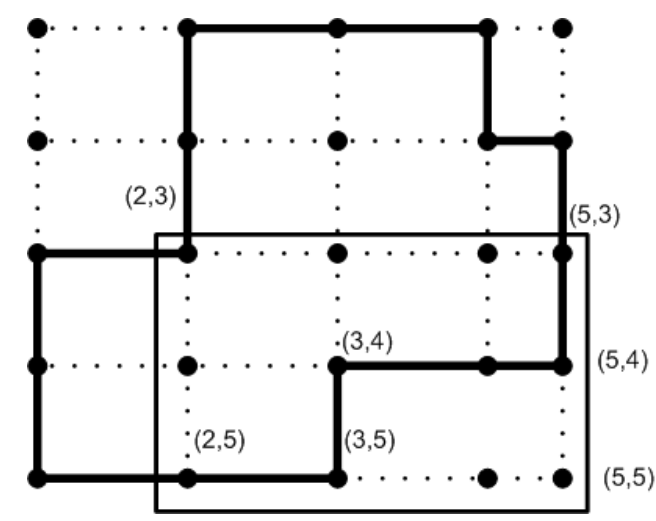

Figure 7: A representation of a sub-rectangle.

1. Sub-rectangles are entirely exterior to the polygon input - Cost 0 .

2. Sub-rectangles are entirely interior to the polygon and are not external slivers - Cost 1 .

3. Sub-rectangles are entirely interior to the polygon and are external slivers - Cost $1+\lambda_{L} L($ exposed edge).

4. Sub-rectangles that consist of both exterior and interior points of the polygon - Determined recursively 
The sub-rectangles that satisfy the first case have zero cost as they do not contain any portion of the input polygon and have no bearing on the resulting fracture. Their existence comes about as an artifact of the representation. In the second and third cases, the sub-rectangle exists completely within the polygon and corresponds to a possible rectangle in the fracture. Therefore their cost contributes to the metric in the following way: 1 to represent the increase in shots and $\lambda_{L} L($ exposed edge) to correspond to an external sliver. These values have been chosen as an extension of the metric developed in Equation (1). Finally, the last case has no immediately obvious cost. We solve for this cost by using the recursive algorithms described in the following two sections.

\subsection{Solving a Cartesian Convex Polygon with a Natural Recurrence}

For an input polygon that has Cartesian convexity, the first step is to form the basic grid and assign a cost to all the sub-rectangles that are readily available. The costs for the remaining sub-rectangles are solved for recursively.

To develop this recurrence we first define a guillotine cut for a given Cartesian convex polygon as a ray that starts from a concave corner and continues until it reaches a boundary of the polygon. Such a ray splits the polygon in half. We also define a rectangle cut to be a cut in a Cartesian direction that splits a rectangle into two smaller rectangles. Finally, a guillotine cut in the polygon is equivalent to a rectangle cut in the embedding rectangle, as shown in Fig. 8.

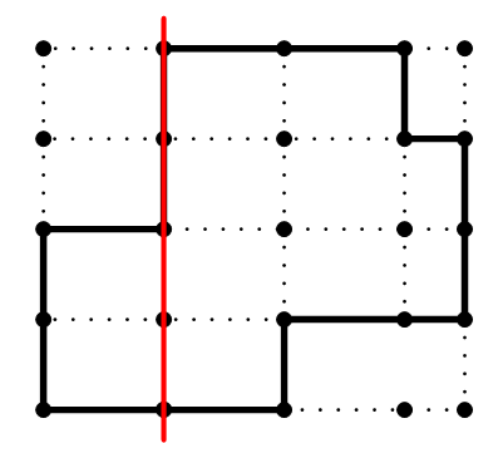

Figure 8: A guillotine cut in the polygon is equivalent to a rectangle cut.

We now describe the recurrence that finds the smallest cost fracturing formed with only guillotine cuts: we take advantage of the fact that a guillotine cut for a Cartesian convex polygon is 
equivalent to a rectangle cut. The recurrence relationship for the sub-rectangles that satisfy case 4 in Section 4.2 can be written as:

$$
\begin{gathered}
F([(a, b),(c, d)])=\min \{ \\
\min _{j \in\{a, \ldots, c\}}[F([(a, b),(j, d)])+F([(j, b),(c, d)])], \\
\min _{k \in\{b, \ldots, d\}}[F([(a, b),(c, k)])+F([(a, k),(c, d)])] \\
\}
\end{gathered}
$$

where $[(a, b),(c, d)]$ is a sub-rectangle and $F([(a, b),(c, d)])$ refers to the optimal fracturing of the subsection of the input polygon embedded by the sub-rectangle defined by $[(a, b),(c, d)]$. The equation states that the optimal fracturing of the sub-rectangle $[(a, b),(c, d)]$ is found by finding the cut that minimizes the cost. This cut is found be sweeping through all horizontal, $j \in\{a, \ldots, c\}$, and vertical, $k \in\{b, \ldots, d\}$, cuts. The recurrence is demonstrated in Fig. 9, where Figs. 9(a), 9(b), and 9(c) show the sweeping of the vertical guillotine cuts and Figs. 9(d), 9(e), and 9(f) show the sweeping of the horizontal guillotine cuts. Each sub-figure in Fig. 9 demonstrates one of the possible decompositions. The algorithm selects the decomposition with the minimal cost. In order to do so, the algorithm may repeat the recurrence for the resulting components of the decomposition.

The above recurrence, by construction, solves for the best fracture that is formed only with guillotine rays. Using dynamic programming the algorithm may be implemented in $O\left(n^{5}\right)$; there are $O\left(n^{4}\right)$ possible sub-rectangles; for each of the sub-rectangles there are $O(n)$ possible cuts that must be considered. Thus, to find the cost of all the sub-rectangles the algorithm requires $O\left(n^{5}\right)$ time where $n$ is the number of vertices.

\subsection{Solving a Cartesian Convex Polygon with an Optimal Recurrence}

In this section we show that the natural recurrence is not always optimal. Fig. 10 shows a possible fracturing that cannot be found by the natural recurrence as no guillotine cuts exist in the fracture. If this fracture is the only optimal one then the natural recurrence finds a sub-optimal solution. 


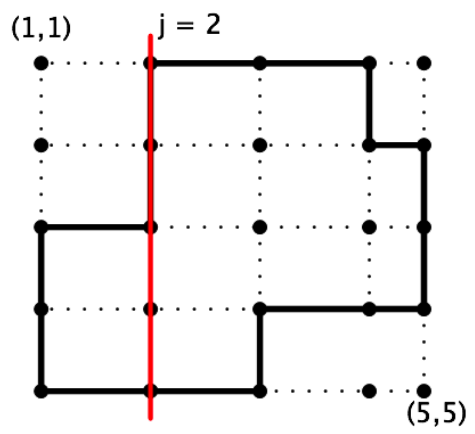

(a)

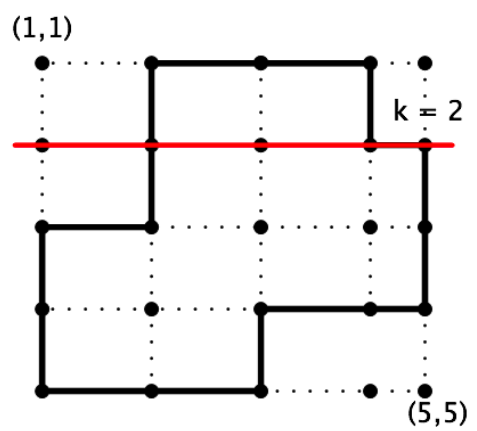

(d)

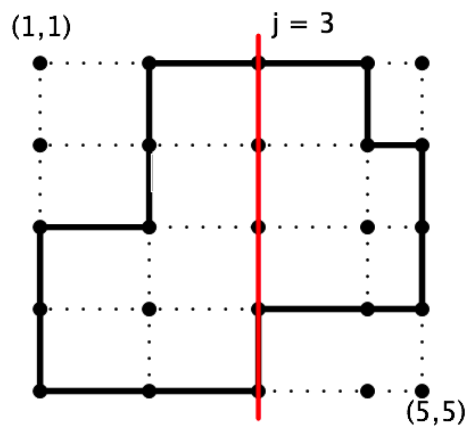

(b)

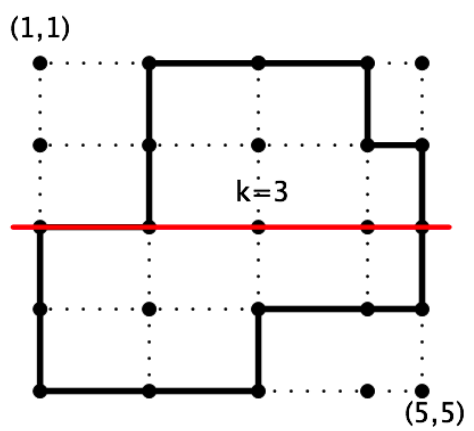

(e)

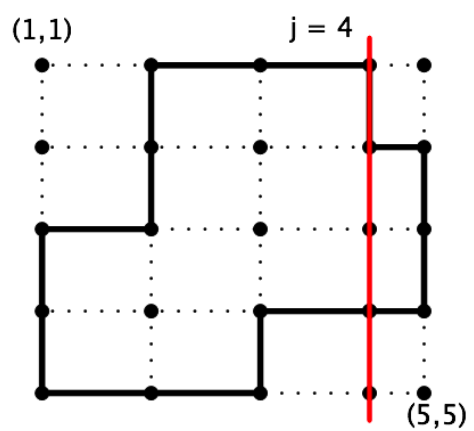

(c)

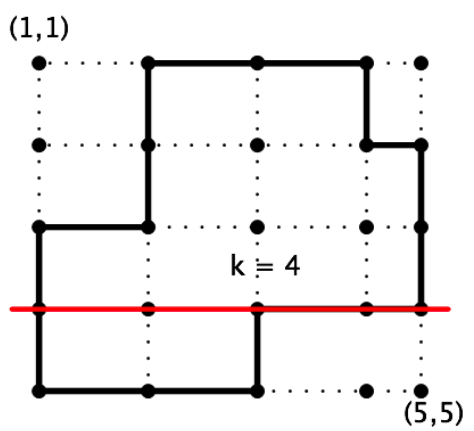

(f)

Figure 9: Decomposition example; vertical guillotine cuts for (a) $j=2$; (b) $j=3$; (c) $j=4$ and horizontal guillotine cuts for (d) $k=2$; (e) $k=3$; (f) $k=4$.

Nevertheless, in Appendix A we show that this configuration is the only possible one in which guillotine cuts fail to fracture a Cartesian convex polygon and use this to develop another algorithm. The first step is to again form the basic grid and assign costs to all the sub-rectangles that are readily available. The remaining costs will be solved for recursively.

Fig. 11 shows the only two possible ways for a decomposition to occur with no guillotine cuts. Both cases are generated by first partitioning the inner rectangle, $[(j, k),(l, m)]$, and then partitioning the remaining shape without guillotine cuts. Fig 11(a) shows case 1, in which the cuts are selected to form a pinwheel rotating clockwise, while Fig 11(b) shows case 2 with a pinwheel rotating counter-clockwise. Again, this implies a recurrence, given below: 


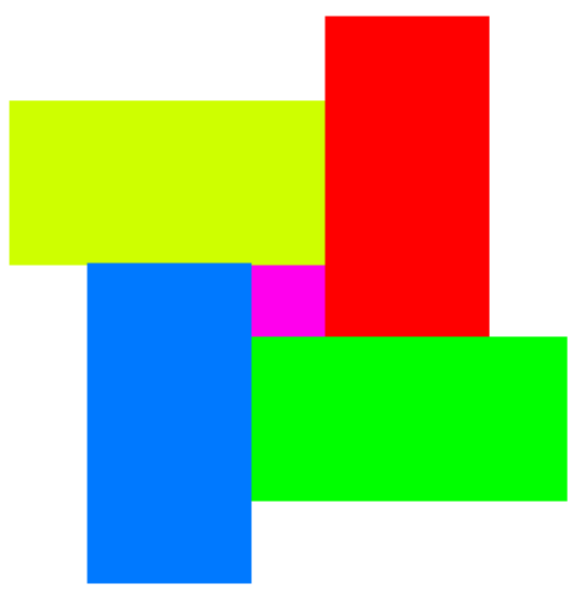

Figure 10: Natural recurrence failing.

$$
\begin{gathered}
F([(a, b),(c, d)])=\min _{a \leq j \leq l \leq c, b \leq m \leq k \leq d}\{ \\
\min [F([(a, b),(l, k)])+F([(l, b),(c, m)])+ \\
F([(a, k),(j, d)])+F([(j, m),(c, d)])+ \\
F([(j, k),(l, m)])], \\
\min [F([(a, b),(j, m)])+F([(j, b),(c, k)])+ \\
F([(a, m),(l, d)])+F([(l, k),(c, d)]) \\
F([(j, k),(l, m)])] \\
\}
\end{gathered}
$$

where $[(a, b),(c, d)]$ is a sub-rectangle and $F([(a, b),(c, d)])$ refers to the optimal fracturing of the subsection of the input polygon embedded by the sub-rectangle defined by $[(a, b),(c, d)]$. The first term in Eqn. (3) corresponds to the cost associated with case 1, shown in Fig. 11(a) and the second term to that of case 2 in Fig. 11(b).

The equation states that the optimal fracturing of the sub-rectangle $[(a, b),(c, d)]$ is found by comparing all possible decompositions and selecting the one with minimal cost. A decomposition is generated by first selecting a sub-rectangle, $[(j, k),(l, m)]$, that resides within the initial rectangle. Next, the algorithm generates the two pinwheel recurrences with the remaining shape, 
$(\mathrm{a}, \mathrm{b})$

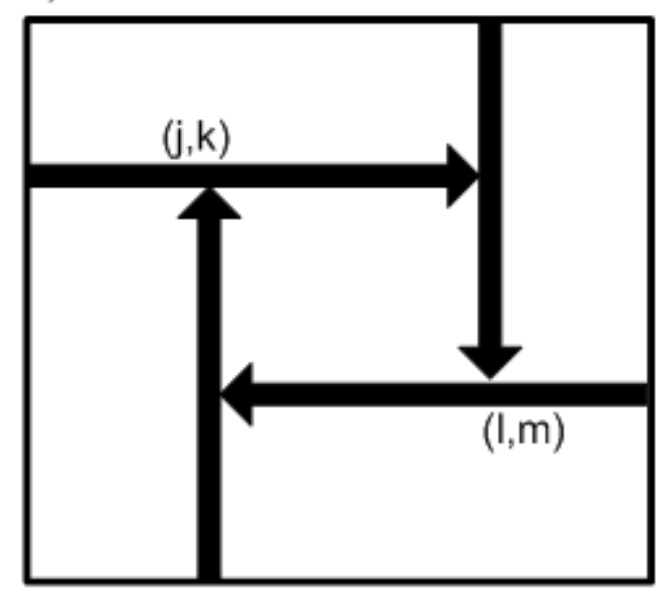

$(\mathrm{c}, \mathrm{d})$

(a) $(a, b)$

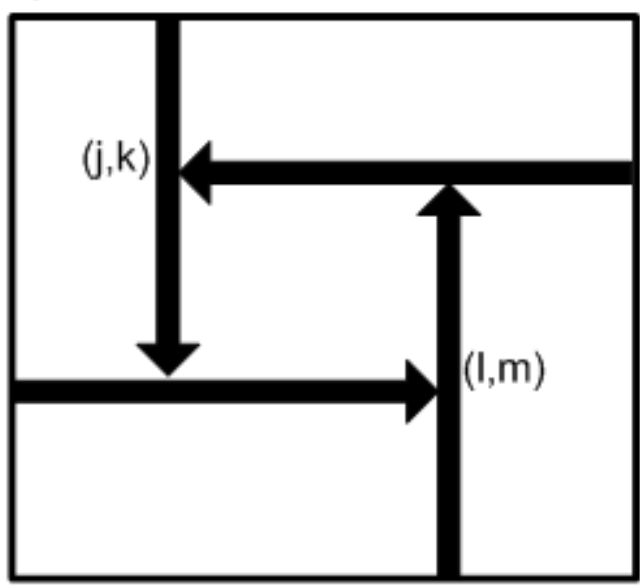

(c,d)

(b)

Figure 11: Fracturing decomposition with no guillotine cuts; (a) Case 1; (b) Case 2.

as shown in Fig 11. This forms one decomposition. The decomposition process is repeated by selecting all sub-rectangles $[(j, k),(l, m)]$ that consist only of points on the basic grid belonging to the strict subset of the input sub-rectangle $[(a, b),(c, d)]$. Upon selecting $[(j, k),(l, m)]$, the same pinwheel recurrence can be generated. After generating all possible decompositions the one with minimal cost is stored as the optimal cost of sub-rectangle $[(a, b),(c, d)]$. The algorithm may need to repeat the recurrence for each resulting decomposition.

The above equation describes a recurrence that does not rely on guillotine cuts. We show that this recurrence is optimal for Cartesian convex polygons and place bounds on the runtime in Appendix A. As explained in Appendix A, the runtime for this recurrence is $O\left(n^{8}\right)$, where $n$ is the number of vertices in the input polygon.

\subsection{Implementation of Recurrences using Dynamic Programming}

The algorithms in Sections 4.3 and 4.4 are recursive, such that a naive implementation would result in an exponential runtime. This is because each sub-rectangle has its cost computed multiple times. A different implementation is provided by dynamic programming. Dynamic programming is a method of solving a complex problem, such as fracturing, by breaking the problem into smaller 
overlapping sub-problems[10]. In particular, it requires the problem to have an optimal structure and the sub-problems to have natural ordering and optimal structure. The first requirement means that the solution to the problem may be given by a combination of optimal solutions for the subproblems. In the fracturing problem this is clear; combining a series of optimal fracturing solutions is equivalent to forming a union of optimal fractures.

The second requirement is that the sub-problems have a natural ordering so that solving a subproblem requires only the solutions to the smaller sub-problems. The fracturing problem satisfies this property in that the optimal fracturing to a sub-rectangle only requires the optimal fracturing to sub-rectangles that are of smaller size. For example, the optimal solution to a sub-rectangle of size $[3 \times 3]$ requires the optimal fracturing to all smaller sub-rectangles: $\{[1 \times 1],[2 \times 1],[3 \times 1]$, $[1 \times 2],[2 \times 2],[3 \times 2],[1 \times 3],[2 \times 3]\}$.

In Sections 4.3 and 4.4 we describe two recurrence expressions to break the fracturing problem into several smaller fracturing sub-problems. Each of these sub-problems correspond to a subrectangle of the basic grid. Next, we solve the sub-problems starting with the smallest. Upon finding the solution to a sub-problem, we store the value in a table. This technique is called memoization[10]. When the cost of a sub-rectangle is needed again, the cost is simply looked up within the table. This limits the runtime to be the product of the number of sub-rectangles with the total number of possible decompositions for each sub-rectangle; as each are polynomial bounded versus the number of vertices, the two recursive algorithms can be computed in polynomial time.

\subsection{Nonconvex Polygons}

The two algorithms in Sections 4.3 and 4.4 have been developed for Cartesian convex polygons. The second algorithm guarantees an optimal fracturing for a Cartesian convex input polygon. In general a layout polygon may not be convex. For the non-convex case we suggest two possible approaches, built upon the above algorithms.

The first approach is the inclusion of a pre-processing step that makes appropriate decisions to split each non-convex polygon into smaller convex polygons. This may be done based on rules such as selecting chords or partitioning rays that do not introduce external slivers. For the remaining 
convex polygons we can run either of the two proposed algorithms. However, the initial decision process may generate a sub-optimal solution.

The second approach is to simply run one of the algorithms in Sections 4.3 and 4.4 on the nonconvex polygon. The algorithm still generates a fracturing but there are no longer any guarantees on optimality. In Section 5, we show results based on this approach for non-convex polygons.

\section{Experimental Results}

In this section we describe simulation results based on our proposed natural recurrence algorithm of Section 4.3. We take Poly and Metal 1 layers from a random logic circuit with CD =90nm and apply an OPC recipe with various levels of aggressiveness as defined by the fragmentation level using the Calibre ${ }^{\mathrm{TM}}$ edge-based OPC software. For all the resulting post-OPC layers we set the sliver parameter, $\epsilon$, to $25 \mathrm{~nm}$, one quarter of the $\mathrm{CD}$. We run the proposed natural recurrence of Section 4.3 on the OPC layers and set the weight, $\lambda_{L}$ to 0.04 . The total resulting rectangle count and external sliver length are found and compared with the Calibre ${ }^{\mathrm{TM}}$ fracture package and provided in Table 1 and 2 [11].

Table 1 contains the performance comparison between the Calibre ${ }^{\mathrm{TM}}$ fracture package and the proposed algorithm for the Poly layer while Table 2 contains the same comparison for Metal 1. For both tables column 1 describes the edge-based OPC fragmentation parameter. Column 2 contains the number of polygons tested for each setting. Column 3 contains the metric of interest, rectangle count or external sliver length. The rectangle count is the sum of the rectangles and the total external sliver length is the sum of the external sliver lengths. Columns 4 and 5 compare the resulting metrics for the Calibre ${ }^{\mathrm{TM}}$ fracture package with our proposed algorithm, and column 6 provides the corresponding percentage improvement. 
Table 1: Comparison of rectangle count and external sliver length between Calibre ${ }^{\mathrm{TM}}$ software and the proposed algorithm for Poly layer with varying OPC fragmentation parameter and $\epsilon=25 \mathrm{~nm}$.

\begin{tabular}{|c|c|c||c|c|c|}
\hline $\begin{array}{c}\text { Fragmentation } \\
\text { Parameters }\end{array}$ & $\begin{array}{c}\text { Polygons } \\
\text { Tested }\end{array}$ & $\begin{array}{c}\text { Fracturing } \\
\text { Metrics }\end{array}$ & $\begin{array}{c}\text { Calibre } \\
\text { Software }\end{array}$ & $\begin{array}{c}\text { Proposed } \\
\text { Algorithm }\end{array}$ & $\begin{array}{c}\text { Percentage } \\
\text { Reduction }\end{array}$ \\
\hline \hline $30 \mathrm{~nm}$ & 310 & Rectangle count & 10183 & 10033 & 1 \\
\hline $30 \mathrm{~nm}$ & 310 & Total external sliver length & 7610 & 6610 & 13 \\
\hline \hline $50 \mathrm{~nm}$ & 325 & Rectangle count & 6614 & 6556 & 1 \\
\hline $50 \mathrm{~nm}$ & 325 & Total external sliver length & 625 & 240 & 62 \\
\hline \hline $70 \mathrm{~nm}$ & 320 & Rectangle count & 5727 & 5651 & 1 \\
\hline $70 \mathrm{~nm}$ & 320 & Total external sliver length & 240 & 120 & 50 \\
\hline
\end{tabular}

Table 2: Comparison of rectangle count and external sliver length between Calibre ${ }^{\mathrm{TM}}$ software and the proposed algorithm for Metal 1 layer with varying OPC fragmentation parameter and $\epsilon=25 \mathrm{~nm}$.

\begin{tabular}{|c|c|c||c|c|c|}
\hline $\begin{array}{c}\text { Fragmentation } \\
\text { Parameters }\end{array}$ & $\begin{array}{c}\text { Polygons } \\
\text { Tested }\end{array}$ & $\begin{array}{c}\text { Fracturing } \\
\text { Metrics }\end{array}$ & $\begin{array}{c}\text { Calibre } \\
\text { Software }\end{array}$ & $\begin{array}{c}\text { Proposed } \\
\text { Algorithm }\end{array}$ & $\begin{array}{c}\text { Percentage } \\
\text { Reduction }\end{array}$ \\
\hline \hline $30 \mathrm{~nm}$ & 165 & Rectangle count & 5022 & 4983 & 1 \\
\hline $30 \mathrm{~nm}$ & 165 & Total external sliver length & 1925 & 1460 & 24 \\
\hline \hline $50 \mathrm{~nm}$ & 389 & Rectangle count & 11371 & 11285 & 1 \\
\hline $50 \mathrm{~nm}$ & 389 & Total external sliver length & 3680 & 3070 & 17 \\
\hline \hline $70 \mathrm{~nm}$ & 201 & Rectangle count & 6429 & 6363 & 1 \\
\hline $70 \mathrm{~nm}$ & 201 & Total external sliver length & 4830 & 2960 & 39 \\
\hline
\end{tabular}

Our proposed algorithm outperforms Calibre ${ }^{\mathrm{TM}}$ for all cases with respect to both metrics. For the Poly layer, when the OPC fragmentation parameter is $30 \mathrm{~nm}$, our algorithm results in a $13 \%$ improvement to external sliver length. For larger values of the OPC fragmentation parameter, there are few slivers. Despite this, our algorithm does a reasonable job to decrease external sliver length by $62 \%$ and $50 \%$ for the OPC fragmentation parameter set to $50 \mathrm{~nm}$ and $70 \mathrm{~nm}$, respectively. In all three cases, our proposed algorithm also lowers the total rectangle count. An example of the performance comparison between Calibre ${ }^{\mathrm{TM}}$ and the proposed algorithm is illustrated in Fig. 
12. The results are generated for Poly layer with the OPC fragmentation parameter set to $30 \mathrm{~nm}$. Fig. 12(a) is the fracture solution of Calibre ${ }^{\mathrm{TM}}$ with 11 rectangles and 70nm of external sliver length. Fig. 12(b) is the fracture solution of the proposed algorithm with 11 rectangles and 40nm of external sliver length. The red lines represent the external slivers.

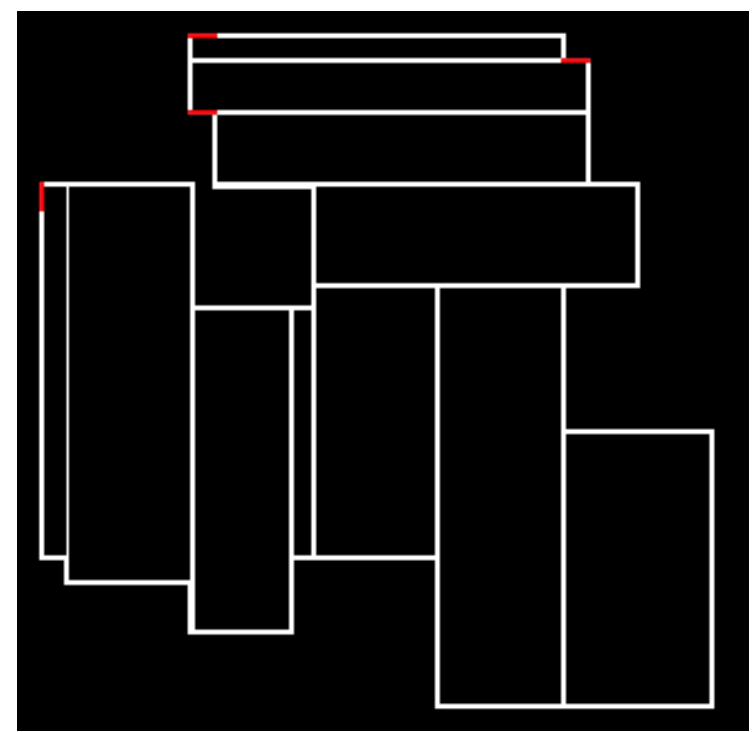

(a)

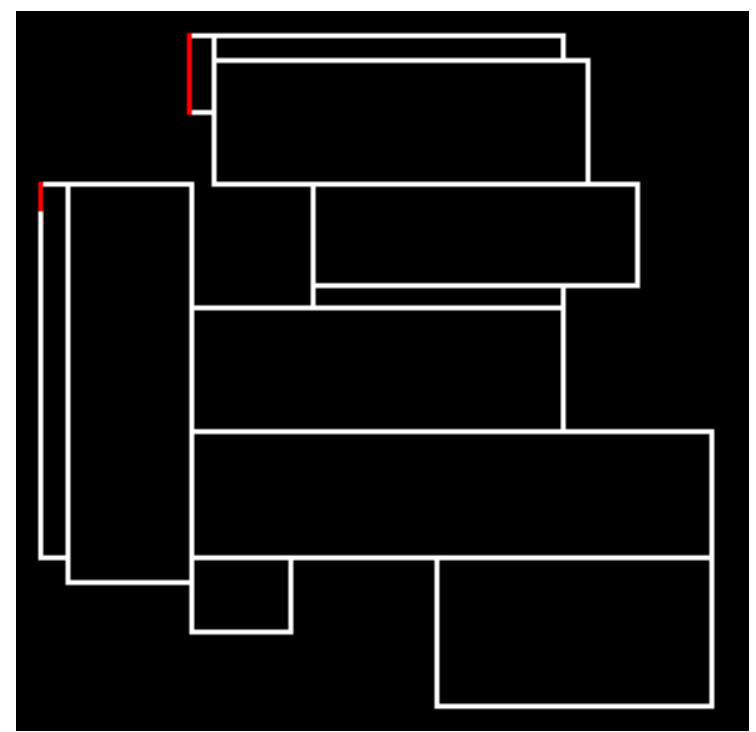

(b)

Figure 12: A performance comparison of Calibre ${ }^{\mathrm{TM}}$ and the proposed algorithm for Poly: (a) Fracture solution generated by Calibre ${ }^{\mathrm{TM}}$ with 11 rectangles and $70 \mathrm{~nm}$ of external sliver length; (b) Fracture solution generated by the proposed algorithm with 11 rectangles and 40nm of external sliver length.

For Metal 1, our proposed algorithm results in about 39\% improvement in external sliver length for when the OPC fragmentation parameter is $70 \mathrm{~nm}$. When the OPC fragmentation parameter is set to $50 \mathrm{~nm}$, it results in only a $17 \%$ improvement in external slivers. However, for all three OPC fragmentation settings there is decreased external sliver length and polygon count. An example of the performance comparison between Calibre ${ }^{\mathrm{TM}}$ and the proposed algorithm is illustrated in Fig. 13. The results are generated for Poly layer with the OPC fragmentation parameter set to $30 \mathrm{~nm}$. Fig. 13(a) is the fracture solution of Calibre ${ }^{\mathrm{TM}}$ with 9 rectangles and 40nm of external sliver length. Fig. 13(b) is the fracture solution of the proposed algorithm with 10 rectangles and no external sliver length. Again, the red lines represent the external slivers.

Our algorithm is able to decrease the external sliver length and number along with the total 
rectangle count. Our algorithm accomplishes this without greatly increasing the total sliver count. In fact, total sliver number barely increased, going from 651 to 718 for Poly layer and going down from 506 to 479 for Metal 1.

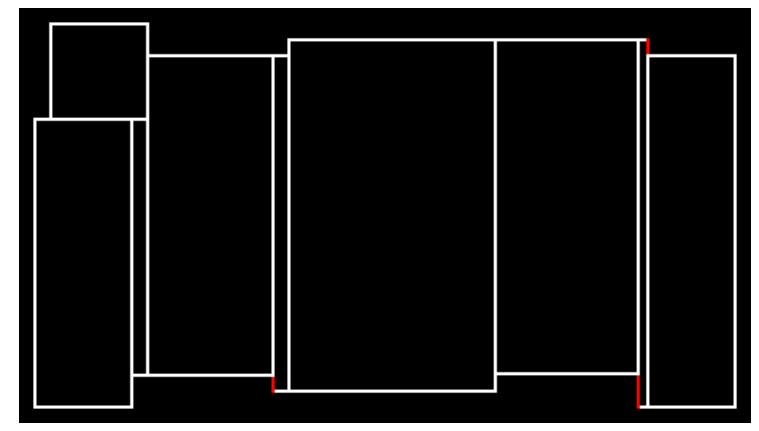

(a)

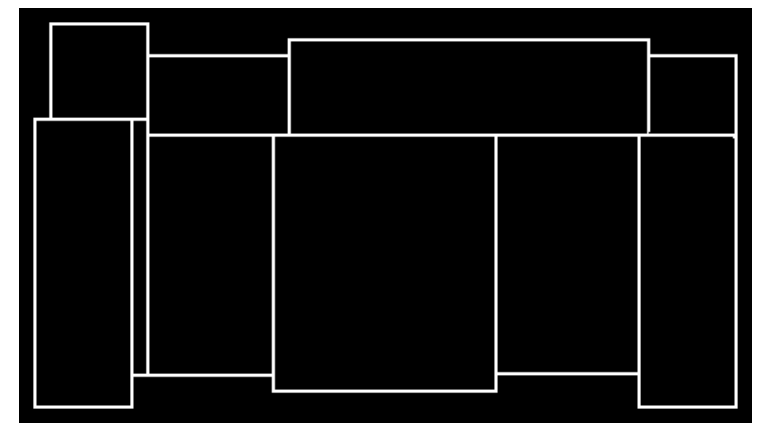

(b)

Figure 13: A performance comparison of Calibre ${ }^{\mathrm{TM}}$ and the proposed algorithm for Metal 1: (a) Fracture solution generated by Calibre ${ }^{\mathrm{TM}}$ with 9 rectangles and 40nm of external sliver length; (b) Fracture solution generated by the proposed algorithm with 10 rectangles and no external sliver length.

Finally, we test our proposed algorithm against the theoretical minimum rectangle count. This is done by running our recursive algorithm with $\lambda_{L}$ parameter set to 0 . For the theoretical result we compute the number of concave corners and maximum number of disjoint chords. This gives us the theoretical minimum number of rectangles needed to properly partition a rectilinear polygon. Our proposed achieves the theoretical minimum as compared with the disjoint chord method for all the 30 tested polygons.

\section{Conclusion and Future Work}

In this paper, we have developed a novel fracturing algorithm based upon recursion and implemented using dynamic programming. First, the polygon is surrounded by a rectangle and all relevant points for testing fracture validity are generated. Second, we compute the cost for all sub-rectangles that are immediately possible. Finally, we compute the cost of the remaining subrectangles using recursion and memoization. This generates the resulting cost and fracture solution. We have shown that for convex rectilinear polygons, a version of the algorithm generates 
an optimal solution. Experimentally, the results for the non-convex case show improvement versus commercially available software tools such as Calibre ${ }^{\mathrm{TM}}$. In fact, simulations show that our algorithm significantly reduces total external sliver length by up to $39 \%$ while not impacting the rectangle count for Metal 1 when the OPC fragment is set to $70 \mathrm{~nm}$. By controlling $\lambda_{L}$, the algorithm is able to achieve the theoretical minimum rectangle count. Both of these facts are promising; the algorithm can both achieve the minimum rectangle count and a decreased external sliver length by controlling a user parameter.

In our future work we plan to expand our cost function to better model fracturing requirements. In particular, we need to include factors such as aspect ratio of rectangles, area of rectangles, and maximum shot size. Second, we need to incorporate certain heuristics to improve runtimes and examine the consequential change to the cost and runtime. Third, we plan to generalize our algorithm, currently only applicable to rectangles, to incorporate trapezoids.

Finally, we plan to investigate other possibilities for the recurrence that may result in better

runtimes and optimality bounds. This is related to our representation; we wish to remove the extra points in the grid that belong to the exterior of the polygon. We plan to base this improvement upon prior work on minimum edge length rectangular partition[12]. This would improve storage needs and would result in faster runtimes.

\section{Acknowledgments}

This research is conducted with funding from Intel.

I would like to thank my research advisor, Professor Avideh Zakhor, for all her guidance. I would like to credit Professor Krste Asanovic and Yunsup Lee for providing the layouts tested in this project. I am grateful to Brian Richards at the Berkeley Wireless Research Center for providing software tools necessary to test the algorithm. 


\section{A Proof of optimality for the pinwheel recurrence}

In this appendix we show that the pinwheel recurrence described in Section 4.4 is optimal for Cartesian convex polygons. First, we define a boundary segment as a line that either is a guillotine cut or part of the polygon boundary. The only possibility for a convex rectilinear polygon to be fractured without a guillotine cut is if the ray $A$ does not terminate on a boundary segment, $B$. However, if $B$ is a non-boundary segment then it must terminate at another non-boundary segment $C$. Similarly, $C$ must terminate at a non-boundary segment, $D$. Because each of these rays involve a $90^{\circ}$ turn, $D$ must terminate at $A$. This implies only the following two options, shown in Fig. 11, exist if a convex rectilinear polygon is not fractured by boundary rays.

Consider temporarily that the rays did not form this sort of pinwheel structure. There are two possibilities for this to occur. The first possibility is if a ray, $A$, needs to be terminated elsewhere, shown in Fig. 14. However, as shown, ray $B$ is no longer be part of the pinwheel structure and could be replaced with $C$. This means the proposed counter-example is identical to the pinwheel recurrence.

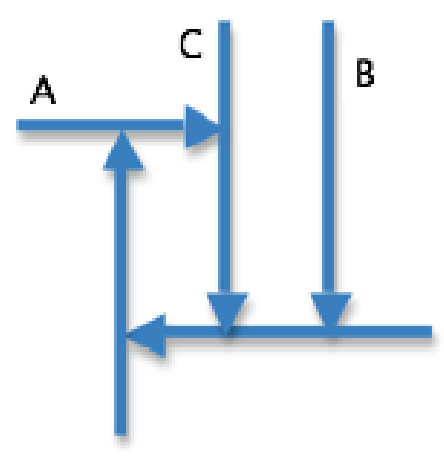

Figure 14: Possible incorrect counterexample 1.

The other possibility is shown in Fig. 15. In this case $A$ could not exist as it does not start at a concave corner. This fact is only true for convex rectilinear polygons. The diagram implies $B$ either passes through the vertex that $A$ starts or goes through a point between $A$ and the starting vertex. In the first case, one of the two rays is unneeded, as both serve to remove the same vertex while in the second case, $A$ is no longer connected to a vertex and thus unneeded.

This shows that the pinwheel recurrence is optimal for the Cartesian convex case. It can be 


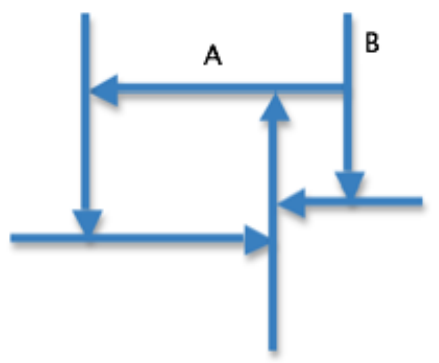

Figure 15: Possible incorrect counterexample 2.

calculated in $O\left(n^{8}\right)$ : there are $O\left(n^{4}\right)$ possible sub-rectangles and each sub-rectangle has $O\left(n^{4}\right)$ possible decompositions. Thus to calculate the cost for all sub-rectangles requires $O\left(n^{8}\right)$ runtime. 


\section{References}

[1] A. B. Kahng, X. Xu, and A. Zelikovsky, "Yield- and cost- driven fracturing for variable shaped-beam mask writing," in Proc. 24th BACUS Symposium on Photomask Technology and Management, pp. 360-371, Sep.(2004)

[2] A. B. Kahng, X. Xu, and A. Zelikovsky, "Fast yield driven fracture for variable shaped beam mask writing” Proc. SPIE Int. Soc. Opt. Eng. 6283, 62832R (2006)

[3] N. Hiroomi, K. Moriizumi, K. Kamiyama, "A new figure fracturing algorithm for high quality variable-shaped EB exposure-data generation”, Proc. SPIE, Kawasaki 1996, 2793:398-409.

[4] C. Spence, S. Goad, P. Buck, R. Gladhill, R. Cinque, J. Preuninger, U. Griesinger, M. Bloecker, "Mask data volume: historical perspective and future requirements, Proc. SPIE Int. Soc. Opt. Eng. 6281, 62810H (2006)

[5] M. Bloecker, R. Gladhill, P. D. Buck, M. Kempf, D. Aguilar, R. B. Cinque, "Metrics to Assess Fracture Quality for Variable Shaped Beam Lithography” Proc. of SPIE Vol. 6349, 63490Z (2006)

[6] T. Ohtsuki, M. Sato, M. Tachibana and S. Torii, "Minimum fracturing of composite rectangular region," Trans. Inf. Process 24, pp. 647-653, (1983)

[7] X. Cheng, D. Du, J. Kim, L. Ruan, “Optimal Rectangular Partitions”, Handbook of Combinatorial Optimization. Ed. Ding-Zhu Du and Panos M. Pardalos. Springer US, (2006). E-Book

[8] D. Kõnig, “Gráfok és alkalmazásuk a determinánsok és a halmazok elméléter” Matematikai és Természettudományi Értesítõ 34: 104-119 (1916)

[9] J. E. Hopcroft, R. M. Richard. "An $n^{5 / 2}$ algorithm for maximum matchings in bipartite graphs”, SIAM Journal of Computing 2 (4) 225-231. (1973)

[10] Bellman, Richard, Dynamic Programming, Princeton University Press. (1957)

[11] http://www.mentor.com/

[12] A. Lingas, R. Y. Pinter, R. L. Rivest, and A. Shamir, "Minimum edge length partitioning of rectilinear polygons", Allerton Conference on Communication, Control, and Computing, (1982) 